\title{
A phosphorothioate antisense oligodeoxynucleotide specifically inhibits coxsackievirus B3 replication in cardiomyocytes and mouse hearts
}

Ji Yuan, Paul KM Cheung, Huifang Zhang, David Chau, Bobby Yanagawa, Caroline Cheung, Honglin Luo, Yinjing Wang, Agripina Suarez, Bruce M McManus and Decheng Yang

Department of Pathology and Laboratory Medicine, The James Hogg iCAPTURE Centre, University of British Columbia-St Paul's Hospital, Vancouver, British Columbia, Canada

\begin{abstract}
Antisense oligodeoxynucleotides (AS-ODNs) are promising therapeutic agents for the treatment of virusinduced diseases. We previously reported that coxsackievirus B3 (CVB3) infectivity could be inhibited effectively in HeLa cells by phosphorothioate AS-ODNs complementary to different regions of the $5^{\prime}$ and $3^{\prime}$ untranslated regions of CVB3 RNA. The most effective target is the proximal terminus of the $3^{\prime}$ untranslated region. To further investigate the potential antiviral role of the AS-ODN targeting this site in cardiomyocytes (HL-1 cell line), corresponding AS-ODN (AS-7) was transfected into the HL-1 cells and followed by CVB3 infection. Analyses by RT-PCR, Western blotting and plaque assay demonstrated that AS-7 strongly inhibits viral RNA and viral protein synthesis as compared to scrambled AS-ODNs. The percent inhibitions of viral RNA transcription and capsid protein VP1 synthesis were 87.6 and 40.1, respectively. Moreover, AS-7 could inhibit ongoing CVB3 infection when it was given after virus infection. The antiviral activity was further evaluated in a CVB3 myocarditis mouse model. Adolescent A/J mice were intravenously administrated with AS-7 or scrambled AS-ODNs prior to and after CVB3 infection. Following a 4-day therapy, the myocardium CVB3 RNA replication decreased by $68 \%$ and the viral titers decreased by $0.5 \log _{10}$ in the AS-7-treated group as compared to the group treated with the scrambled AS-ODNs as determined by RT-PCR, in situ hybridization and viral plaque assay. Taken together, our results demonstrated a great potential for AS-7 to be further developed into an effective treatment towards viral myocarditis as well as other diseases caused by CVB3 infection.
\end{abstract}

Laboratory Investigation (2004) 84, 703-714, advance online publication, 19 April 2004; doi:10.1038/labinvest.3700083

Keywords: antisense; cardiomyocyte; coxsackievirus B3; mouse; myocarditis

Coxsackievirus B3 (CVB3) is a member of the genus Enterovirus of the family Picornaviridae. ${ }^{1}$ It can infect multiple organs of humans and certain animals, and causes myocarditis, pancreatitis, meningitis and other diseases. According to the global surveillance of viral diseases conducted by the World Health Organization, CVB3 is the most common cause of viral myocarditis, which may lead to the development of dilated cardiomyopathy and heart failure in the late stage of infection. ${ }^{2-4}$ To date, there are no specific antiviral treatments existing towards this important pathogen.

CVB3 is a single-stranded positive-polarity RNA virus with a genome of approximately $7.4 \mathrm{~kb}$ in

Correspondence: Dr D Yang, Cardiovascular Research Laboratory, University of British Columbia, St Paul's Hospital, 1081 Burrard Street, Vancouver, BC, Canada V6Z 1 Y6.

E-mail: dyang@mrl.ubc.ca

Received 16 September 2003; revised 9 December 2003; accepted 16 December 2003; published online 19 April 2004 length. It has a long $5^{\prime}$ untranslated region ( $\left.5^{\prime} \mathrm{UTR}\right)$ containing 741 nucleotides but a short $3^{\prime}$ UTR (100 nucleotides) with a polyadenyl (poly-(A)) tail at the $3^{\prime}$ end. It is believed that the $3^{\prime}$ UTR, containing an intramolecular kissing interaction between two hairpin loops, is important for regulating picornavirus RNA replication. ${ }^{5-7}$ Deletion of the entire $3^{\prime}$ UTR of poliovirus and rhinovirus results in low infectivity and poor replication. ${ }^{8}$ In addition, interactions between the viral $3^{\prime}$ UTR and certain cellular proteins have been reported to play crucial roles in viral RNA replication and possibly in viral translation. For example, mutations in the $3^{\prime}$ UTR of poliovirus, coxsackievirus and rhinovirus can decrease cellular protein-binding activity, which in turn decreases RNA replication and virus viability. ${ }^{9}$ Therefore, efficient blocking of the $3^{\prime}$ terminal region of CVB3 RNA may inhibit the binding of cellular translation or transcription initiation factors, thereby preventing the formation of a replication unit of a circular viral RNA. ${ }^{10}$ 
The potential of oligodeoxynucleotides to act as antisense agents that inhibit the replication of the Rous sarcoma virus was discovered in $1978 .{ }^{11}$ As a novel class of therapeutic agents to selectively block or inhibit gene expression, antisense oligodeoxynucleotides (AS-ODNs) have attracted great interest in the development of drugs and gene functional studies. They have been widely tested for the treatment of infections, cancers and inflammatory diseases. Several of these studies have reached the stage of clinical trials, and one antisense drug Vitravene (Formivirsen) was approved by the Food and Drug Administration. ${ }^{12}$ Because of the small and simple nature of the viral genome as compared to other organisms, AS-ODN antiviral strategies have great potential to achieve specific inhibition. In the past years, the antiviral effects of AS-ODNs have been demonstrated in a number of different viruses such as influenza A virus, ${ }^{13}$ human immunodeficiency virus, ${ }^{14}$ hepatitis B virus ${ }^{15-17}$ and foot-andmouth disease virus. ${ }^{18}$ However, the effectiveness of unmodified AS-ODNs is limited by the low efficiency of cellular uptake and the lack of stability due to degradation by nucleases. To become nuclease resistant, phosphorothioate (PS) AS-ODNs have been produced by substitution of one of the nonbridging oxygen atoms at the phosphorus with sulfur. In vivo pharmacokinetic and distribution studies of PS AS-ONDs of various sequences have been reported in several animal models by using radioisotope-labeled ODNs and FITC-ODNs. ${ }^{19-22}$ These studies found that PS AS-ONDs were distributed to all major organs, including the liver, kidney and heart, and were taken up by cells following intravenous administration. ${ }^{23}$

Previously, to investigate the specific antiviral activities of AS-ODNs, seven PS AS-ODNs, complementary to different regions of the $5^{\prime}$ and $3^{\prime}$ UTRs of CVB3 RNA, were designed and synthesized based on our former mutational mapping of the cis- and trans-acting translational sequence elements and evaluated in HeLa cells. ${ }^{7,24,25}$ We showed that the PS AS-7 targeting the proximate terminus of the $3^{\prime}$ UTR has the strongest antiviral activity in HeLa cells. ${ }^{26}$ Since CVB3 is a cardiotropic virus and commonly induces viral myocarditis, in this study we further evaluated AS-7 in mouse cardiomyocytes and in the hearts of $\mathrm{A} / \mathrm{J}\left(\mathrm{H}-2^{\mathrm{a}}\right)$ mouse, an established CVB3 myocarditis model. We have demonstrated that AS7 possesses specific anti-CVB3 activity in both HL-1 cells and mouse hearts. This suggests that AS-7 is a promising candidate for the further development of an effective treatment for CVB3 infection.

\section{Materials and methods}

\section{Synthesis of AS-ODNs}

The design and synthesis of AS-ODNs have been previously described. ${ }^{26}$ Three AS-ODNs were synthesized for this study. One is the specific ODN
(AS-7, 5' CCGCACCGAATGCGGAGAAT 3') targeting the proximal terminus of $3^{\prime} \mathrm{UTR}$ of CVB3 RNA. The other two are control ODNs: one is the corresponding scrambled sequence (AS-7S, 5' ACGACGTCGATCGAACGACG $3^{\prime}$ ), which has the same length and GC content as the AS-7; the other is the general scrambled sequence (AS-S, 5' ACGTTGCAACGTCGTATCAT $3^{\prime}$ ) containing the same length and the average GC content of the full-length CVB3 genome RNA. These ODNs were synthesized by the standard phosphoramidite chemistry method using an Applied Biosystems DNA/RNA synthesizer on a $1 \mu \mathrm{M}$ scale at the Biotechnology Laboratory, University of British Columbia. In order to replace the phosphodiester bonds within the oligonucleotides with phosphorothioates, the oxidation step was substituted with a sulfurization procedure using Beaucage's reagents. The oligonucleotide derivatives were then purified by reverse-phase HPLC chromatography, lyophilized and the powder was dissolved in sterile phosphate-buffered saline (PBS). The FITC-labeled PS AS-S (5' end labeled with FITC), which was used for testing the optimal transfection conditions, was purchased from Qiagen.

\section{Virus, Cell Culture and Transfection}

CVB3 stock was generously provided by $\mathrm{Dr}$ Reinhard Kandolf (Germany) and was stored at $-80^{\circ} \mathrm{C}$ until use. The virus was propagated in HeLa cells (American Type Culture Collection), and titers were routinely determined at the beginning of the experiments.

The HL-1 cell line, a cardiac muscle cell line established from an AT-1 mouse atrial cardiomyocyte tumor lineage, was a kind gift from Dr William C Claycomb. ${ }^{27}$ The cells were maintained in Claycomb medium (JRH Biosciences, Lenexa, KS, USA) supplemented with $10 \%$ fetal bovine serum (JRH Bioscience), $100 \mu \mathrm{g} / \mathrm{ml}$ penicillin/streptomycin (Life Technologies), $0.1 \mathrm{mM}$ norepinephrine (Sigma) and $2 \mathrm{mM}$ L-glutamine (Life Technologies).

The transfection of HL-1 cardiomyocytes with ODNs was performed under optimal conditions. Cell density, amount of Oligofectamine ${ }^{\mathrm{TM}}$ (Life Technologies) and the AS-ODN concentration were determined by preliminary experiments using FITClabeled PS AS-S. The transfection efficiency was evaluated by counting the number of positive transfected cells under a fluorescent microscope. Briefly, $2 \times 10^{5}$ HL-1 cells seeded in gelatin/fibronectin-coated (Sigma) plates were grown in Claycomb medium at $37^{\circ} \mathrm{C}$ for $20 \mathrm{~h}$. When cells reached approximately $50 \%$ confluency, they were overlaid with transfection complexes containing $10 \mu \mathrm{M}$ ASODN and $10 \mu \mathrm{l}$ of Oligofectamine ${ }^{\mathrm{TM}}$ and incubated for another $4 \mathrm{~h}$. After transfection, the cells were washed with PBS and infected with CVB3 at a multiple of infection (MOI) of 10 for $1 \mathrm{~h}$. Following infection, the cells were overlaid with complete 
medium and incubated for $40 \mathrm{~h}$. For therapeutic experiments, HL-1 cells were infected with CVB3 for $1 \mathrm{~h}$ and then transfected with AS-ODNs. Finally, supernatants from each treatment were collected by centrifugation at $4000 \mathrm{~g}$ for $5 \mathrm{~min}$. The resulting supernatant was aliquoted and stored at $-80^{\circ} \mathrm{C}$ until use.

\section{Viral Infection of Mice}

Thirty 4-week-old A/J [H-2 $\left.{ }^{\mathrm{a}}\right]$ mice (Jackson Laboratory) were randomized to three groups and infected intraperitoneally with $10^{5}$ plaque forming units (PFU) of CVB3. Mice were administrated AS-7 $(n=15)$, AS-7S $(n=5)$ or AS-S $(n=10)(20 \mu \mathrm{g} / \mathrm{g}$ body weight) intravenously $2 \mathrm{~h}$ before infection and days 1, 2, 3 post infection (p.i.). All mice were sacrificed on day 4 p.i. Subsequently, the ventricular portions of the hearts were collected and transversely sectioned into the apex, mid and basal portions for analysis.

\section{Biodistribution of AS-ODN, Histology and In Situ Hybridization}

To determine the distribution of AS-ODN throughout the organs, $20 \mu \mathrm{g} / \mathrm{g}$ body weight of FITC-labeled AS-S was injected into the tail veins of 4 -week-old A/J mice. Control animals were injected with AS-S. All the mice were killed $24 \mathrm{~h}$ after injection. Frozen liver and heart sections were counterstained with $4^{\prime}$, 6-diamidino-2-phenylindole (DAPI, Molecular Probes) and examined by fluorescence microscopy.

For histological analysis, formalin-fixed midventricular portions of cardiac tissue were sectioned into 4- $\mu \mathrm{m}$ slices, and stained with hematoxylin and eosin (H\&E). H\&E sections were graded in a blinded manner for the extent of myocarditis based on the intensity and character of injury and inflammatory infiltration by a cardiac pathologist as previously described. ${ }^{28}$ Grades were based on the following scale: 0 meaning no or questionable presence; 1 for limited focal distribution; 2-3 for intermediate severity; and 4-5 for coalescent and extensive foci over the entirety of the transversely sectioned ventricular tissue.

Further, sections were also used for the detection of viral positive- and negative-stranded RNA by in situ hybridization as previously described. ${ }^{29}$ Briefly, paraffin-embedded tissue sections were permeabilized with proteinase $\mathrm{K}$, dehydrated, and hybridized with a digoxigenin-labeled CVB3 probe prepared by in vitro transcription according to the manufacturer's instructions (Promega). Hybridization occurred at $42^{\circ} \mathrm{C}$ overnight followed by stringent washings in $50 \%$ formamide and $2 \times$ SSC. After an antidigoxigenin antibody was applied to tissue sections, slides were enzymatically developed and then incubated overnight with a color substrate.

\section{RT-PCR}

For in vitro evaluation, viral RNA was extracted from $100 \mu \mathrm{l}$ of supernatant using TRIZOL reagent (Gibco-BRL). Extracted RNA was dissolved in $30 \mu \mathrm{l}$ of diethyl pyrocarbonate-treated water. For in vivo studies, total RNA was extracted from the basal portion of the ventricular tissues with an RNease kit (Qiagen). Reverse transcription (RT) was conducted according to the manufacturer's instructions (Invitrogen) using $30 \mu \mathrm{l}$ of RNA (in vitro) or $1 \mu \mathrm{g}$ of extracted RNA (in vivo) and $1 \mu \mathrm{l}$ of $3 \mu \mathrm{M}$ hexamer primer and followed by PCR to amplify CVB3 cDNA (nt 8-601). The PCR reaction mixture contained $10 \mu \mathrm{l}$ of RT product and $1 \mu \mathrm{l}$ of $15 \mu \mathrm{M}$ sense and antisense primers, and the reaction was run for 25 cycles within standard parameters. For in vivo evaluation, $\beta$-actin served as the control. In all $20 \mu \mathrm{l}$ of PCR product from each sample was analyzed by $0.8 \%$ agarose gel electrophoresis. The bands were quantified by densitometry scanning and the density of CVB3 cDNA bands were normalized with that of $\beta$-actin. To determine the antiviral activity of ASODN, the statistically analyzed means and standard error (s.e.) of the band densities were normalized with respect to the control, whose mean value was converted to 100 .

\section{Western Blot}

In total, $20 \mu \mathrm{l}$ of supernatant from each ODN treatment was analyzed for the CVB3 structural protein, VP1, by Western blot as described previously. ${ }^{26}$ Briefly, the supernatants were denatured at $95^{\circ} \mathrm{C}$ for $10 \mathrm{~min}$ and the proteins were separated by SDS-polyacrylamide gel electrophoresis. The samples were then transferred to a nitrocellulose membrane at $75 \mathrm{~V}$ for $60 \mathrm{~min}$. The membranes were blocked with PBS-Tween 20 solution (PBS-T) containing 5\% skimmed milk at room temperature for $2 \mathrm{~h}$, and then incubated with primary rabbit antiVP1 IgG (gift from Dr R Kandolf) at $4^{\circ} \mathrm{C}$ overnight. The membranes were washed with PBS-T for $30 \mathrm{~min}$ and followed by incubation with a secondary antibody (goat anti-rabbit IgG conjugated to horseradish peroxidase, Santa Cruz). The signal detection occurred through the enhanced chemiluminescence method according to the manufacturer's instructions (Amersham Pharmacia Biotech, Inc., USA). All experiments were performed three times. The exposed films were analyzed by densitometric scanning of the bands. Quantitation of the VP1 was performed by the methods described above for PCR products.

\section{Viral Plaque Assay}

Antiviral activity of the AS-ODN was determined by plaque assay for both in vitro and in vivo studies. In the evaluation with HL-1 cells, CVB3 titers were 
determined using the supernatants collected from the AS-ODN-treated cardiomyocytes. For evaluation using mouse model, apex portions of the ventricules were weighed and then homogenized in Dulbecco's modified Eagle medium (Gibco-BRL); after three cycles of freeze-thawing to release intracellular viruses, the supernatant was collected by centrifugation at $4000 \mathrm{~g}$ for $5 \mathrm{~min}$ and used for plaque-forming assay following the method described previously ${ }^{29}$ with minor modifications. Briefly, HeLa cells were seeded into six-well plates $8 \times 10^{5}\left(8 \times 10^{5}\right.$ cells/ well) and incubated at $37^{\circ} \mathrm{C}$ for $20 \mathrm{~h}$. When cell confluency reached approximately $90 \%$, cells were washed with PBS to remove the fetal bovine serum and then overlaid with $1 \mathrm{ml}$ of 1:10 diluted supernatant. The cells were incubated at $37^{\circ} \mathrm{C}$ for $60 \mathrm{~min}$ and the supernatants were removed, and followed by a PBS wash. Finally, cells were overlaid with $2 \mathrm{ml}$ of sterilized soft Bacto-agar/MEM $(2 \times$ MEM: $1.5 \%$ Bacto-agar $=1: 1)$. The cells were incubated at $37^{\circ} \mathrm{C}$ for $72 \mathrm{~h}$, fixed with Carnoy's fixative for $30 \mathrm{~min}$ and then stained with $1 \%$ crystal violet. The plaques were counted and the viral pfu/ml was calculated.

\section{Statistical Analysis}

The results are expressed as means \pm s.e. Statistical analyses were performed with unpaired Student's $t$ test. $P$ values less than 0.05 were considered to be statistically significant.

\section{Results}

\section{Inhibition of CVB3 Replication in Mouse Cardiomyocytes}

We reported previously that CVB3 infectivity could be inhibited effectively in HeLa cells by AS-ODNs complementary to different regions of the $5^{\prime}$ and $3^{\prime}$ UTRs. One of the most effective AS-ODNs is the AS-7 targeting the proximal terminus of the $3^{\prime} \mathrm{UTR}$.
To further investigate the inhibitory effect of AS-7 on CVB3 replication in mouse cardiomyocytes, the HL-1 cell line, which can be serially passaged and yet maintains a cardiac-specific phenotype, ${ }^{27}$ was employed for in vitro evaluation of the inhibitory activity of AS-7. In addition, this cell line has been shown to express the coxsackievirus-adenovirus receptor and is susceptible to CVB3 infection although it requires a 10 -fold higher MOI to obtain a similar level of infection as that in HeLa cells (C Cheung, unpublished data).

To reach high transfection efficiency in HL-1 cells, different cell density, varying amounts of Oligofectamine $^{\mathrm{TM}}$ and AS-ODN concentration were examined using FITC-AS-S. The optimal transfection efficiency for HL-1 cells was approximately $30 \%$ as determined by calculation of the ratio of fluorescent cells to total cells under a fluorescence microscope (data not shown). Also, cell confluency at approximately $50 \%$ is the optimal condition for transfection. Meanwhile, to obtain the most significant inhibitory effects under AS-ODN treatments, different MOIs of CVB3 and dosages of AS-ODNs were evaluated as described previously. ${ }^{26}$ We found that MOI of CVB3 at 10 and concentration of AS-ODN at $10 \mu \mathrm{M}$ were optimal conditions for evaluation of the drugs in HL-1 cells.

Following these conditions, HL-1 cells were first transfected with AS-7, scrambled AS-7S or general scrambled AS-S, and then infected with CVB3. At $40 \mathrm{~h}$ p.i., the antiviral activity of AS-7 was evaluated by comparing the cell morphology and death rate of cultures pretreated with AS-7 against that of two controls. Figure 1 demonstrates that infected cells in AS-7-treated cultures were healthier and larger in size than control AS-ODN-treated cells, which seemed unhealthy as most cells appeared rounded or floating. The percentage of living cells in the AS7-treated group and controls that were all infected with CVB3 were approximately 60 and $20 \%$, respectively (the two controls showed a similar rate of cell death). However, the cells treated with AS-
AS-S

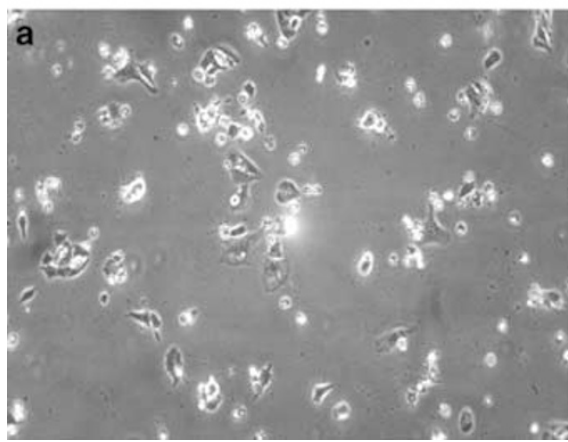

AS-7S

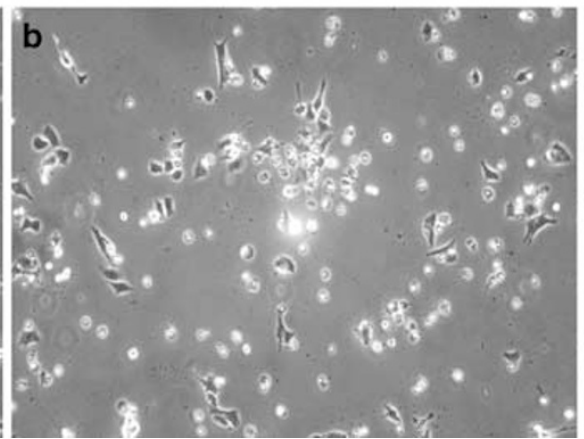

AS-7

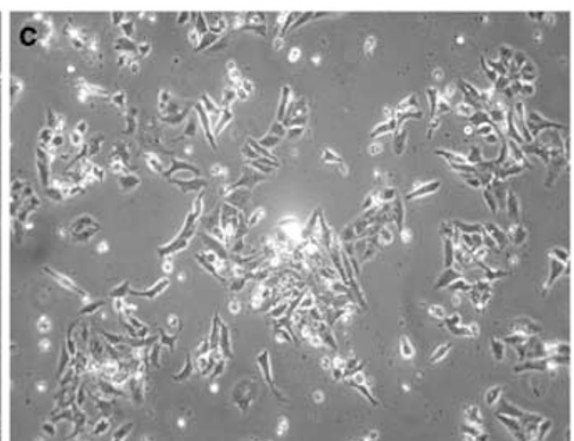

Figure 1 AS-7 inhibits CVB3-induced cell death. HL-1 cells were transfected with AS-ODN at an final concentration of $10 \mu \mathrm{M}$ for $4 \mathrm{~h}$ and then infected with CVB3 at an MOI of 10. Cellular morphology was observed under a phase contrast microscope $40 \mathrm{~h}$ p.i. $(\times 100)$. Cells treated with AS-7 contain more viable cells than the other two controls treated with AS-S or AS-7S, in which the majority of the cells are rounded and floating. 
ODNs only were mostly healthy and viable, indicating that AS-7 is not toxic to HL-1 cells (data not shown).

In further analysis, we evaluated the antiviral effect of AS-7 on CVB3 RNA transcription in HL-1 cells. Viral genomic RNA was amplified by RT-PCR using supernatants collected from the different ASODN-treated cultures. The PCR products were quantified by densitometric scanning of the bands. To compare the antiviral action of AS-7 to the two control AS-ODNs, the statistically analyzed means and s.e. of the densities were normalized by a value, which converted the mean of the AS-S group to 100 . Figure 2a shows that AS-7 has an $87.6 \%$ inhibitory effect on CVB3 RNA replication.

The antiviral activity of AS-7 in HL-1 cells was also evaluated by Western blot analysis of viral capsid protein VP1 production. Supernatants collected from CVB3-infected cultures pretreated with AS-ODNs were separated by SDS-polyacrylamide gel electrophoresis, and viral capsid protein VP1 was detected using a recombinant rabbit anti-VP1 antibody. Since CVB3 RNA encodes a single long polyprotein including structural and nonstructural proteins, the synthesis of structural protein VP1 fully represents CVB3 translation efficiency. In order to determine the antiviral activity of AS-7, the density values of CVB3 VP1 bands were measured and normalized as described for RT-PCR product. Figure 2b illustrates that AS-7 shows strong inhibitory effects on VP1 synthesis. Compared with the two controls, the percent inhibition of CVB3 VP1 synthesis by AS-7 is $40.1 \%$.

To estimate infectious viral particle production following AS-ODN treatments, plaque assay was performed to measure the viral titer in the supernatant. Viral plaques were counted 3 days after overlaying soft agar on a HeLa cell monolayer. The numbers of $\mathrm{PFU} / \mathrm{ml}$ were calculated. Figure 2c demonstrates that AS-7 strongly inhibited the production of infectious CVB3 particles and that the viral titer in the AS-7-treated group was $0.5 \log _{10}$ lower than that in the control-treated cultures. This result is consistent with the data obtained by RTPCR and Western blot.

Figure 2 AS-7 inhibits CVB3 replication in HL-1 cells. (a) RT-PCR analysis of viral RNA. Supernatant from each sample was used to detect CVB3 genomic RNA by RT-PCR. The PCR bands were quantified by densitometry. The value of the control AS-S was normalized to a value of 100 . The values of AS-7S and AS-7 were calculated with respect to the AS-S value and expressed in the upper panel. (b) Western blot analysis of CVB3 capsid protein VP1. HL-1 cells were transfected with AS-ODN by Oligofecta$\operatorname{mine}^{\mathrm{TM}}$ for $4 \mathrm{~h}$ and then infected with CVB3. At $40 \mathrm{~h}$ p.i., the cellfree supernatant of each sample was collected for the viral protein VP1 detection by Western blot. Quantitation of the products was conducted by the method described above for viral RNA. (c) Plaque assays of infectious viral particles. The differences between the values for AS-7 and the control, AS-S or AS-7S, were statistically significant $\left(P<0.05\right.$, marked as $\left.{ }^{*}\right)$. The lower panel is a representative plate of the plaque assay.
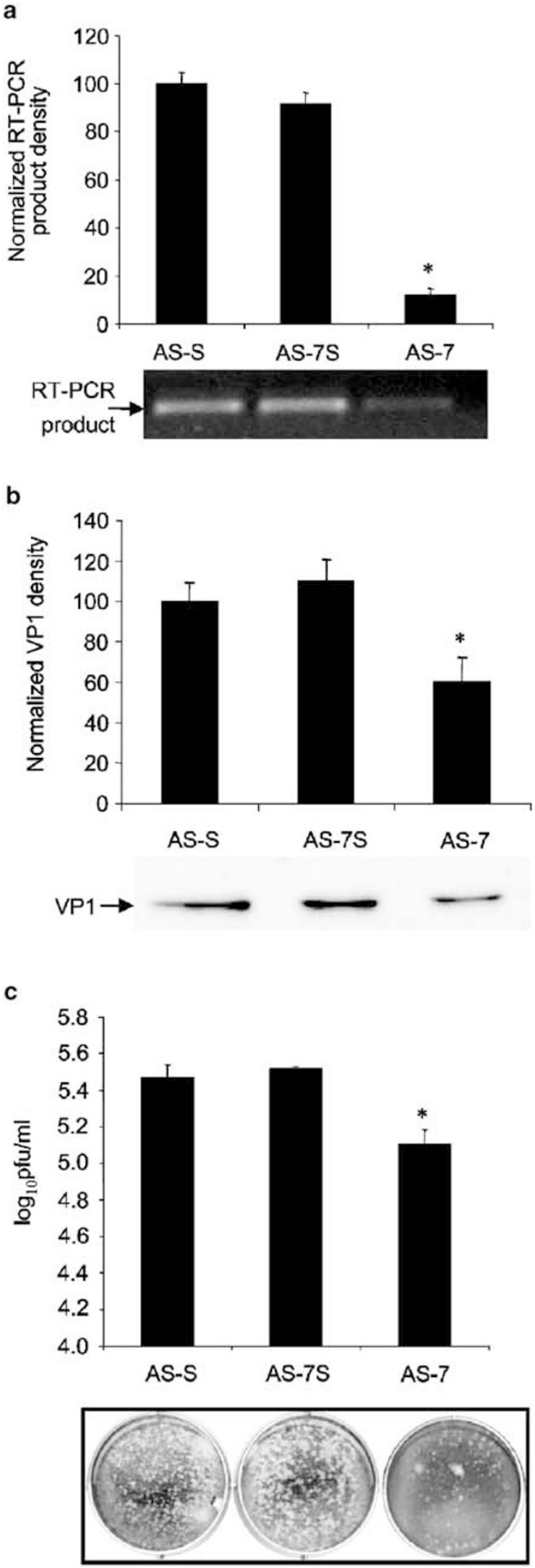


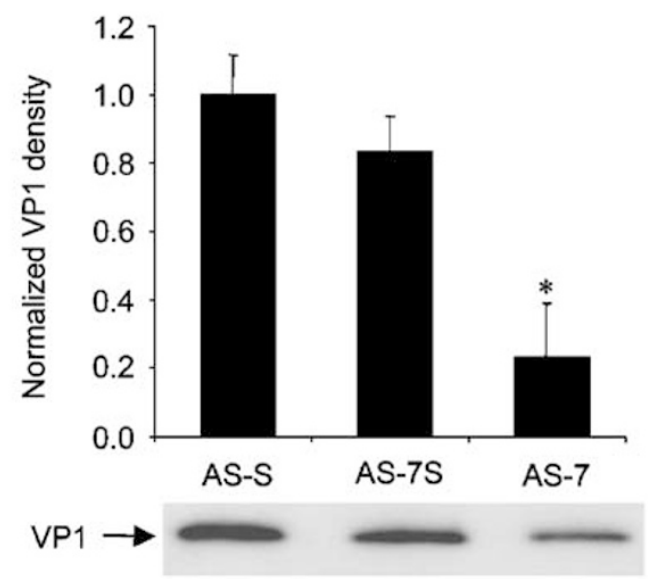

b
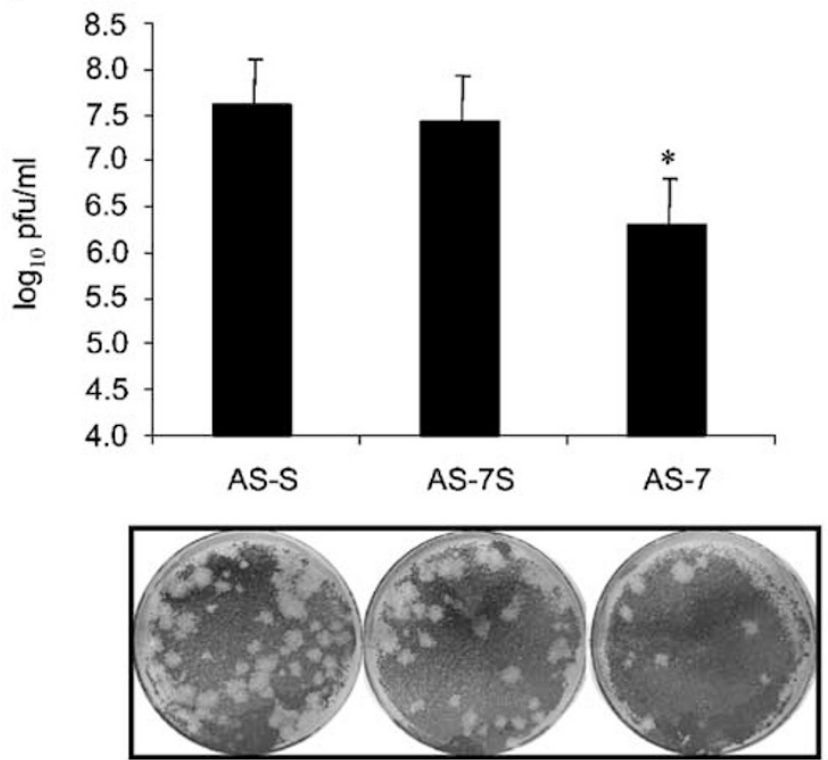

Figure 3 AS-7 inhibits ongoing CVB3 replication in HL-1 cells. HL-1 cells were infected with CVB3 at an MOI of 10 for $1 \mathrm{~h}$ and were then transfected with AS-ODNS separately. At $40 \mathrm{~h}$ following infection, supernatants were collected for detecting viral protein VP1 and infectious virus particles. (a) Western blot analysis of CVB3 capsid protein VP1. Quantitation of the products was conducted by the method described above. (b) Plaque assays of infectious viral particles. The differences between the values for AS-7 and the control, AS-S or AS-7S, were statistically significant $\left(P<0.05\right.$, marked as $\left.{ }^{*}\right)$. The lower panel is a representative plate of the plaque assay.

Considering clinical applications in viral myocarditis, AS-ODNs must be able to effectively inhibit an ongoing virus infection. To further evaluate its therapeutic potential, HL-1 cells were infected with CVB3 for $1 \mathrm{~h}$ and then transfected with AS-ODNs. Supernatants were collected at $40 \mathrm{~h}$ p.i. for detecting viral protein VP1 expression and virus titer. As shown by Western blot (Figure 3a) and viral plaque assay (Figure $3 \mathrm{~b}$ ), viral protein VP1 expression as well as viral titer were significantly reduced by AS-7 treatment. Thus, administration of AS-7 after viral infection can also inhibit viral replication effectively.

\section{Inhibition of CVB3 Replication in Mouse Hearts}

Based on the in vitro studies, we further evaluated the antiviral activity of AS-7 in vivo. First, to test whether the PS AS-ODN could be delivered into the living myocardium, FITC-labeled AS-S at $20 \mu \mathrm{g} / \mathrm{g}$ of body weight was injected intravenously and hearts and livers were harvested at $24 \mathrm{~h}$ post-treatment to investigate the cellular internalization of AS-ODN by fluorescence microscopy. Previous in vivo pharmacokinetic studies reported that oligonucleotides were distributed to all major organs, with liver and kidney accumulating most of the oligonucleotides after systemic administration. ${ }^{23}$ Thus, we used liver as our positive control for the evaluation of distribution. As shown by counterstaining in Figure 4, fluorescent AS-S could be delivered into the cardiomyocytes and other cardiac cells, although the density is lower than that in liver. Such observations concur with previous reports. ${ }^{23}$ Based on these preliminary experiments, AS-7, AS-S or AS-7S was administrated intravenously prior to and following CVB3 infection. At $24 \mathrm{~h}$ after the last treatment, clinical signs of illness, such as weight loss, decreased activity and roughed fur, were similar in all three groups with a range of weight loss from 19.6 to $20.5 \%$. Up to 4 days p.i., only one mouse, whose body weight was lower than others, died at 2 days p.i. in the AS-7 group.

In determining the anti-CVB3 effect of AS-7 treatments in mouse hearts, several evaluatory experiments, including quantification of viral RNA and viral titer as well as comparison of myocarditis severity in the hearts following AS-ODN treatments, were performed. As mentioned in the in vitro evaluation, RT-PCR was conducted for CVB3 RNA quantification using total RNA extracted from the basal portion of the ventricle, the area sensitive to CVB3 infection. Figure 5a shows that the viral genome RNA products in the hearts treated with AS-7 were $68.0 \%$ less than those treated with AS-S or AS-7S. The RT-PCR results were further confirmed by in situ hybridization (Figure 5b). To further quantify the viral progeny production in the myocardium, viral titers were measured by plaque assay using the apex portion of the ventricle. As shown in Figure 5c, the titer of infectious virus particles in the AS-7-treated group is $0.5 \log _{10}$ less than that in control groups treated with either AS-S or AS-7S. The severity of myocarditis as determined by the degree of cellular injury and inflammation was graded without a knowledge of the experimental groups. At day 4 p.i., although the hearts of all groups were characterized by small disseminated foci of inflammation, the average grades of AS-7 group were lower than that of the other two control groups (Figure 6). However, such differences did not reach statistical significance $(P>0.05)$. This suggests that the severity of myocarditis may not be proportional to the viral loading in the heart within certain levels or periods of virus infection. 


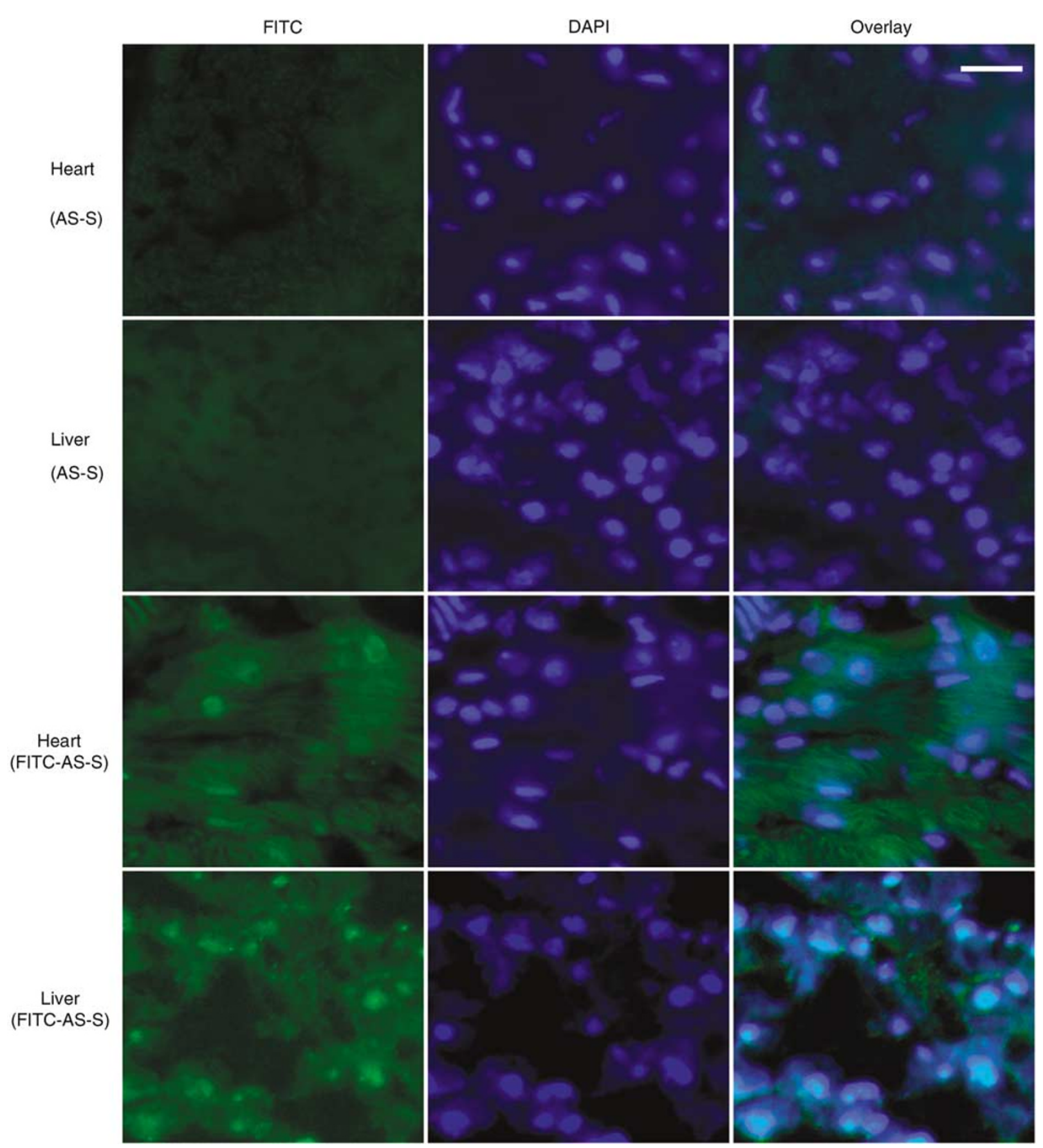

Figure 4 Distribution of FITC-labeled AS-ODN in murine hearts. FITC-labeled AS-S was administrated by intravenous injection of A/J mice. Control animals were injected with AS-S. After $24 \mathrm{~h}$ animals were euthanized and hearts were harvested. Nuclei were counterstained with DAPI. Localization of the FITC-labeled AS-ODNs was observed through fluorescence microscopy $(\times 400)$. Note the fluorescent signals present in the heart and liver treated with FITC-AS-S as compared with the controls treated with AS-S. Scale bar represents $20 \mu \mathrm{m}$.

\section{Discussion}

The AS-ODNs have been widely used in studies of gene function and gene therapy by specific inhibition of certain gene expression. We previously reported that the AS-ODNs, which are complementary to crucial sites of CVB3 RNA, have specific inhibitory effects on both viral protein synthesis and RNA replication in HeLa cells. Clinically, no specific treatments exist for CVB3 infection thus far. As a follow-up study, our aim is to further test the efficacy of AS-ODNs as a therapeutic agent for CVB3 infection in cardiomyocytes and in a wellestablished murine model since CVB3 is a cardiotropic virus and a common pathogen of acute myocarditis. $^{3}$

Experience from this study and others has suggested that two major factors are critical in 
710

a

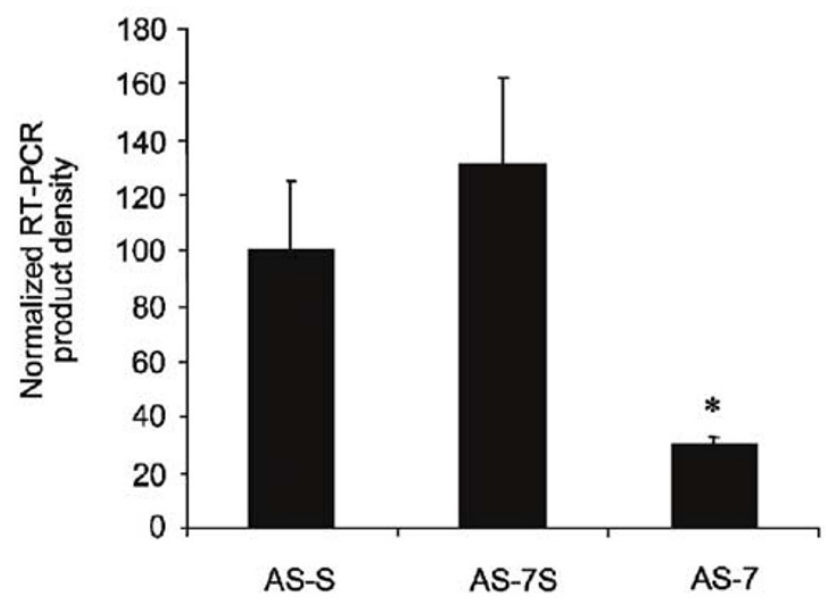

b

AS-S

AS-7S

AS-7

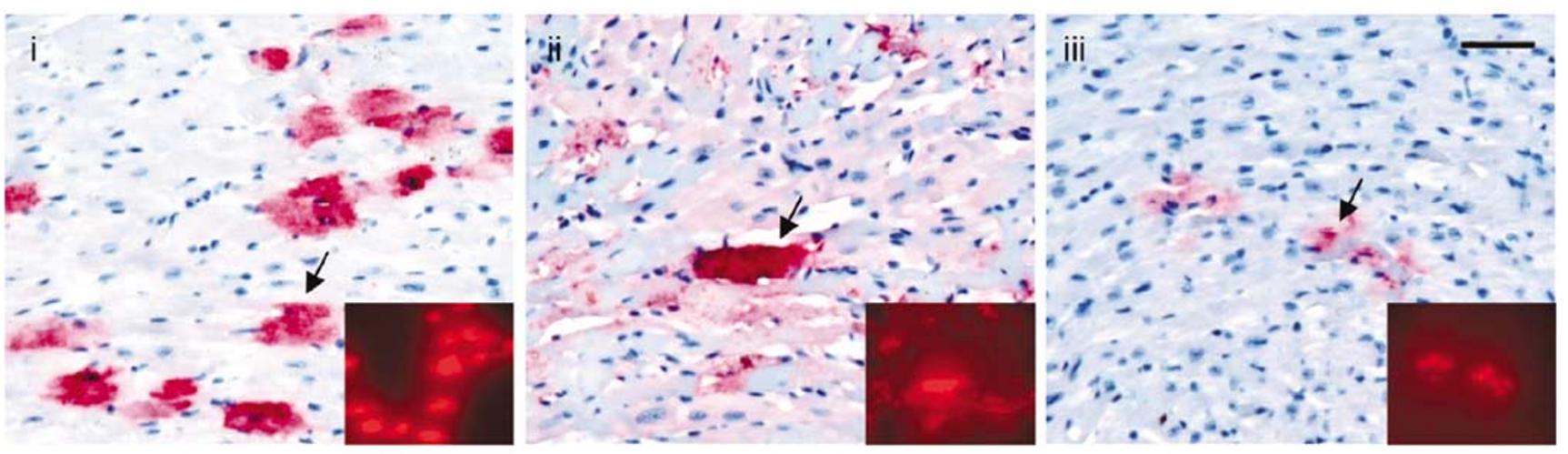

c
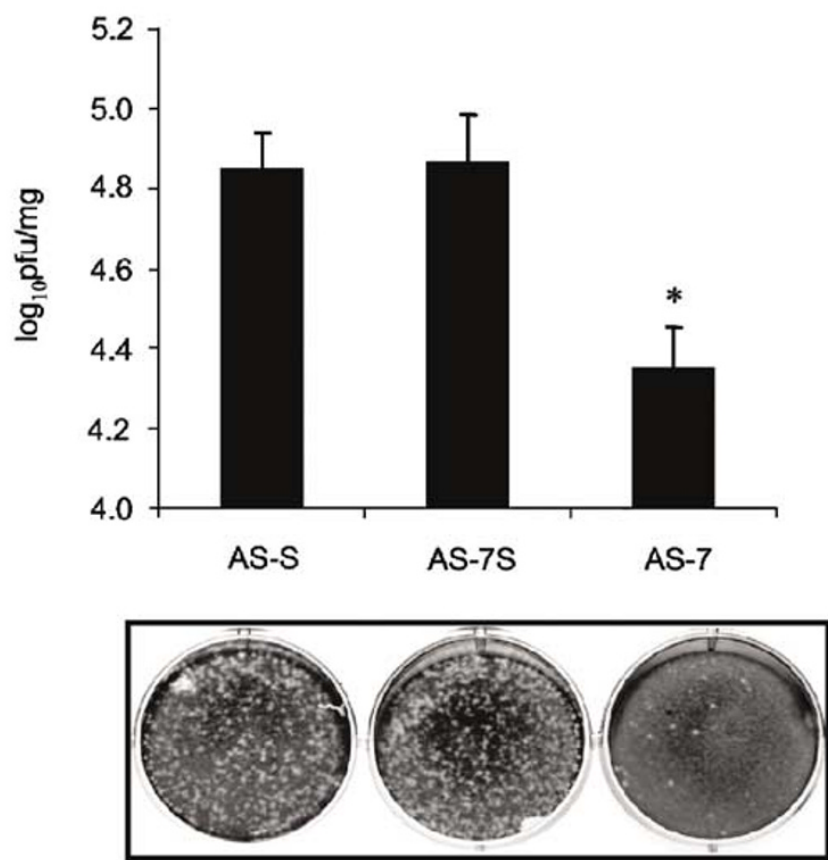
determining the effectiveness of the AS-ODNs. The first is the selection of target sites within the mRNA, in which specific AS-ODNs block gene expression efficiently and selectively. For our case, CVB3 is a single-stranded positive-sense RNA virus, containing a long $5^{\prime} \mathrm{UTR}$ and a short $3^{\prime} \mathrm{UTR}$ flanking the single open reading frame. It has been reported that removal of the entire $3^{\prime} \mathrm{UTR}$ region of poliovirus and rhinovirus results in low infectivity and poor replication. ${ }^{8}$ Further, it is known that the $5^{\prime}$ and $3^{\prime}$ UTRs of CVB3 RNA form highly ordered tertiary

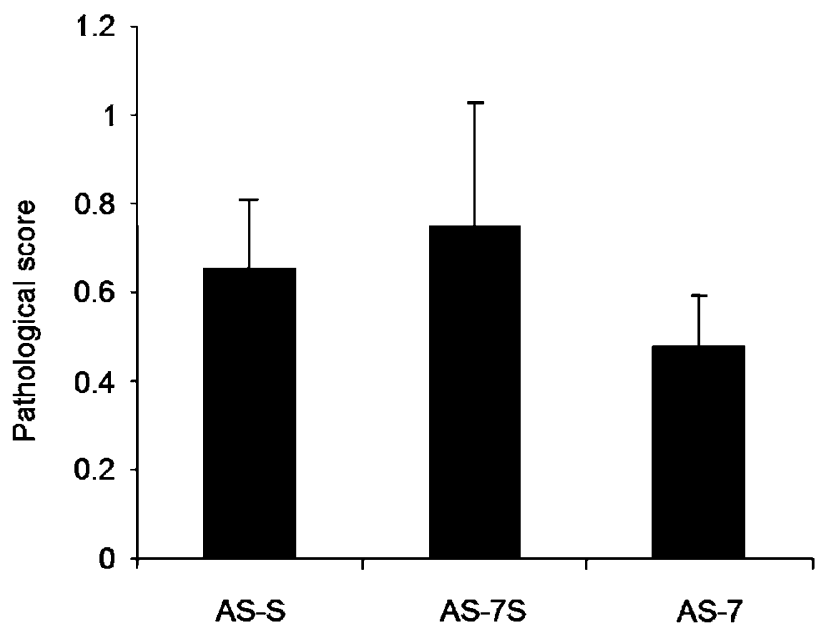

Figure 6 Effect of AS-7 treatment on the severity of CVB3induced myocarditis. Infection and treatments were the same as that described in Figure 4. Mid-portions of ventricular sections were stained with hematoxylin and eosin and severity of the disease was semiquantitatively graded based on the intensity and character of injury and the inflammatory infiltration. Note that disease severity of the AS-7-treated animals is much lower than that of the other two control groups. However, such differences are only close to the statistical significance, indicating that more timepoints need to be evaluated.

Figure 5 AS-7 inhibits CVB3 replication in mouse hearts. (a) RTPCR analysis of CVB3 RNA transcripts. A/J $\left[\mathrm{H}-2^{\mathrm{a}}\right]$ mice were infected intraperitoneally with CVB3 at $10^{5}$ PFU $2 \mathrm{~h}$ after the first administration of AS-ODN and then followed by three additional intravenous injections at days 1,2 and 3 p.i. Mice were killed on day 4 and hearts were collected for total RNA extraction. RT-PCR was conducted using this total RNA as described in the Materials and Methods. The PCR products were quantified as described in the legend to Figure 2. The difference in values between the controls (AS-S or AS-7S) and AS-7 groups is statistically significant $(P<0.05)$. (b) In situ hybridization of CVB3 RNA. The selected sections are representative of in situ hybridization results of the mouse hearts pretreated with AS-S (i), AS-7S (ii) and AS-7 (iii), respectively $(\times 400)$. The images at the right lower corner were taken under dark field. Scale bar represents $50 \mu \mathrm{m}$. (c) Plaque assay of infectious CVB3 particles. The apical portions of the hearts were weighed and homogenized in DMEM. After three cycles of freeze-thawing to release intracellular viruses, the supernatant was used for plaque-forming assays as described in Figure 2c. The differences in values (pfu/mg) between the control (AS-S or AS-7S) and AS-7 prove to be statistically significant $(P<0.05)$, which are presented in the upper panel. The lower panel shows the representative plaques of the assay. structures serving as recognition features for the RNA-binding site of host proteins and have important cis-acting functions in the replication and translation of viral RNA. For example, single nucleotide mutations on the hairpin loops of the $3^{\prime}$ UTR disrupt tertiary interactions, thereby resulting in temperature-sensitive virus. ${ }^{6,25,30-32}$ In addition, our recent protein-3'UTR interaction experiments by UV cross-linking found that certain host proteins specifically interact with the $3^{\prime} \mathrm{UTR}$ of CVB3. Most protein-RNA interactions depend on the existence of the poly-(A) tail sequence, suggesting that most of the binding proteins either interact specifically to the poly-(A) tail or bind to the secondary structures of the $3^{\prime} \mathrm{UTR}$, which can be maintained by the interactions with the poly-(A) sequence ( $\mathrm{P}$ Cheung, unpublished data). From our previous evaluation of AS-ODNs in infected HeLa cells, the highest inhibitory activity among all seven AS-ODNs was obtained with AS-7 targeting the terminus of the $3^{\prime} \mathrm{UTR},{ }^{26}$ which is the site for transcription initiation of negative strands of viral RNA. Moreover, the $5^{\prime}$ - and $3^{\prime}$-UTR end interactions of viral RNA have been shown to be necessary for several viral RNA syntheses. ${ }^{33,34}$ Such interaction is likely mediated by several host cell proteins, such as poly-(A)-binding protein, an important trans-acting factor required for viral translation and replication. ${ }^{10}$ Thus, targeting the terminus of $3^{\prime}$ UTR by AS7 will interfere with $5^{\prime}$ - and $3^{\prime}$-UTR end interactions of viral RNA, which in turn inhibit viral transcription and translation. The data reported here have confirmed that AS-7 confers specific activity in both cardiomyocytes and in the mouse model wherein the pathophysiological conditions are much closer to a clinical environment. Such antiviral ability can be interpreted by the significantly lower viral RNA synthesis, decreased viral capsid protein expression and reduced progeny viral particle formation. There are two possible modes of the AS-7 anti-CVB3 action: interfering with viral RNA synthesis and/or blocking viral RNA translation. However, in this study we cannot distinguish whether AS-7 plays a more important role in viral translation or RNA replication, probably due to the CVB3 special life cycle. The CVB3 genome is a positive-sense singlestranded RNA that functions as both the viral messenger RNA and the template for RNA replication via a negative stranded intermediate. Such a unique life cycle causes translation and replication to interact with each other; therefore, inhibition of either step subsequently affects the other.

The second factor is the instability of unmodified free AS-ODNs and its low nonselective cellular uptake, hindering the therapeutic usefulness in vivo. To resolve this issue, modified PS AS-ODNs, which combine many desired pharmacokinetic properties such as high biostability, RNase $\mathrm{H}$ activation and target specificity, ${ }^{35,36}$ were synthesized and administrated intravenously in vivo. PS AS-ODNs have a half-life in human serum of approximately $9-10 \mathrm{~h}$ as 
compared to $1 \mathrm{~h}$ for unmodified ODNs. ${ }^{37-39}$ Previous in vivo transfer of FITC-labelled AS-ODNs into mouse hearts by direct needle injection demonstrated a half-life of ODN within 1-3 days. ${ }^{40}$ Here, we showed that AS-ODNs lasted for $24 \mathrm{~h}$ postinjection as the FITC-labeled AS-ODN parallel control could be clearly detected in the heart at the same timepoint. Free PS AS-ODNs that we administered by intravenous injection have the advantages of being economical, easy to handle and less toxic than those with synthetic carriers, such as linear polyethylenimine, which has shown toxicity in in vivo applications. ${ }^{41}$ Moreover, in vivo pharmacokinetic and distribution studies have shown that free PS AS-ODNs are well absorbed from parenteral sites and distribute broadly to organs and peripheral tissues via intravenous administration. ${ }^{23,36}$ Further, efficient cellular uptake of AS-ODNs in vivo has been achieved without the use of any delivery system. $^{42}$ Currently, eight PS AS-ODNs are being investigated in phase II and III clinical trials for a broad range of diseases, including viral infections, cancer and inflammatory diseases. ${ }^{42}$ In terms of the way of administration, several studies were previously conducted by direct injection of ODN into the heart. ${ }^{43-45}$ However, this direct injection resulted in a limited area of transfection and localized myocyte toxicity. To avoid this, we used intravenous administration as it is gentle to handle without causing injury of the heart and also makes the drug spread evenly.

In data analysis, we found that several parameters could significantly affect the antiviral efficiency of AS-7 in HeLa cells and in HL-1 cells. For example, the viral replication rate, that is, the time required for a single replication cycle of CVB3, varies according to the different host cell types. In addition, the rate of cellular internalization of the AS-ODN is dependent on the cell type and the medium in which the uptake occurs. Another parameter, the transfection efficiency of AS-ODN in different cells, highly varies even under optimized conditions. We have tested the transfection efficiency in HeLa cells and HL-1 cells, and the optimal transfected rates were approximately 50 and $30 \%$, respectively. This provides one explanation for the stronger antiviral effects of AS-7 in HeLa cells than that in HL-1 cells.

The observed antisense effects are sequence specific since the control AS-ONDs containing a scrambled sequence did not reduce virus translation and replication. It has been reported that synthetic ODNs, which contain specific CpG motifs, mimic bacterial DNA and have been shown to induce a coordinated set of immune responses that comprise innate and acquired Th1-biased cellular and humoral immunity. ${ }^{46-48}$ The concerns were raised because $\mathrm{CpG}$-induced activation of $\mathrm{B}$ cell proliferation and immunoglobulin secretion protects against lethal challenge with a wide variety of pathogens, such as ebolavirus and
Listeria monocytogenes. ${ }^{49,50}$ However, the AS-7 we used in the current study did not contain CpG motifs; thus, the inhibition of virus production through nonspecific immune response would be less likely to occur.

In pathohistological evaluations, the AS-7-treated group resulted in less severe myocarditis as compared to the other two control groups. However, the difference was not significant even though virus titers were significantly lower in AS-7-treated tissue than in control-treated tissues. This may reflect the fact that viral titers in certain ranges may not be proportional to the severity of myocarditis. Similar to many other viral illnesses, both direct viral injury and the immune response of the host play important roles in the pathogenesis of myocarditis. ${ }^{51}$ Some studies showed that viral direct effects occur independently of an immune response, ${ }^{52,53}$ while other in vivo experiments demonstrated that the immune response contributes to both detrimental and beneficial effects on the host. ${ }^{54-58}$ However, the relationship between viral injury and the immune response in myocarditis remains incompletely understood. In this case, it is likely that the immune responses leading to inflammation were comparable only within certain ranges of virus loads and certain timepoints of infections. If the evaluation is performed at later timepoints p.i., the pathological improvement in AS-7-treated animals may be more significant as compared to the control groups. Thus, to address this issue, further studies at different timepoints need to be conducted.

In summary, we have shown that intravenous administration of modified PS AS-ODNs targeting the $3^{\prime}$ end of the $3^{\prime}$ UTR results in a specific and effective inhibition of CVB3 replication both in vitro and in vivo. To our knowledge, this is the first report to evaluate the anti-CVB3 activity of AS-ODNs in an animal model. The significant reduction of CVB3 transcription and translation in the mouse hearts indicates that AS-7 possesses great potential to be developed into an effective therapeutic agent for the treatment of viral myocarditis and other related diseases caused by CVB3 infection.

\section{Acknowledgements}

We thank Dr Reinhard Kandolf, University of Tubingen, Germany, for providing us with CVB3 and Dr William C Claycomb for providing us with HL-1 cells. We thank all the members in the laboratory for their support and help, especially Elizabeth Walker, Zhongshu Luo and Jingchun Zhang. This study was supported by Canadian Institutes of Health Research, Grant \# MOP-14068, and Heart and Stroke Foundation of BC and Yukon, Grant \# 20R20002. 


\section{References}

1 Abelmann WH. Viral myocarditis and its sequelae. Annu Rev Med 1973;24:145-152.

2 Johnson RA, Palacios I. Dilated cardiomyopathies of the adult (first of two parts). $\mathrm{N}$ Engl J Med 1982;307:1051-1058.

3 Hosenpud JD, Novick RJ, Breen TJ, et al. The Registry of the International Society for Heart and Lung Transplantation: Eleventh Official report-1994. J Heart Lung Transplant 1994;13:561-570.

4 McManus BM, Kandolf R. Myocarditis: evolving concepts of cause, consequence, and control. Curr Opin Cardiol 1991;6:418-427.

5 Pilipenko EV, Poperechny KV, Maslova SV, et al. Ciselement, oriR, involved in the initiation of $(-)$ strand poliovirus RNA: a quasi-globular multi-domain RNA structure maintained by tertiary ('kissing') interactions. EMBO J 1996;15:5428-5436.

6 Melchers WJ, Hoenderop JG, Bruins Slot HJ, et al. Kissing of the two predominant hairpin loops in the coxsackie B virus $3^{\prime}$ untranslated region is the essential structural feature of the origin of replication required for negative-strand RNA synthesis. J Virol 1997;71:686-696.

7 Wang J, Bakkers JM, Galama JM, et al. Structural requirements of the higher order RNA kissing element in the enteroviral $3^{\prime}$ UTR. Nucleic Acids Res 1999;27:485-490.

8 Todd S, Towner JS, Brown DM, et al. Replicationcompetent picornaviruses with complete genomic RNA $3^{\prime}$ noncoding region deletions. J Virol 1997;71:8868-8874.

9 Mellits KH, Meredith JM, Rohll JB, et al. Binding of a cellular factor to the $3^{\prime}$ untranslated region of the RNA genomes of entero- and rhinoviruses plays a role in virus replication. J Gen Virol 1998;79(Part 7): 1715-1723.

10 Huang P, Lai MM. Heterogeneous nuclear ribonucleoprotein a1 binds to the $3^{\prime}$-untranslated region and mediates potential $5^{\prime}-3^{\prime}$-end cross talks of mouse hepatitis virus RNA. J Virol 2001;75: 5009-5017.

11 Zamecnik PC, Stephenson ML. Inhibition of Rous sarcoma virus replication and cell transformation by a specific oligodeoxynucleotide. Proc Natl Acad Sci USA 1978;75:280-284.

12 Marwick C. First 'antisense' drug will treat CMV retinitis. JAMA 1998;280:871.

13 Mizuta T, Fujiwara M, Hatta $\mathrm{T}$, et al. Antisense oligonucleotides directed against the viral RNA polymerase gene enhance survival of mice infected with influenza A. Nat Biotechnol 1999;17:583-587.

14 Lisziewicz J, Sun D, Klotman M, et al. Specific inhibition of human immunodeficiency virus type 1 replication by antisense oligonucleotides: an in vitro model for treatment. Proc Natl Acad Sci USA 1992;89:11209-11213.

15 Offensperger WB, Offensperger S, Walter E, et al. In vivo inhibition of duck hepatitis $\mathrm{B}$ virus replication and gene expression by phosphorothioate modified antisense oligodeoxynucleotides. EMBO J 1993;12:1257-1262.

16 Moriya K, Matsukura M, Kurokawa K, Koike K. In vivo inhibition of hepatitis B virus gene expression by antisense phosphorothioate oligonucleotides. Biochem Biophys Res Commun 1996;218:217-223.
17 Dong B, Shao XW, Tao PZ. Inhibition of duck hepatitis $B$ virus DNA replication by antisense phosphorothioate oligodeoxynucleotides in vitro and in vivo. Zhonghua Shi Yan He Lin Chuang Bing Du Xue Za Zhi 2003;17:25-27.

18 Rosas MF, Martinez-Salas E, Sobrino F. Stable expression of antisense RNAs targeted to the $5^{\prime}$ non-coding region confers heterotypic inhibition to foot-andmouth disease virus infection. J Gen Virol 2003;84:393-402.

19 Saijo Y, Perlaky L, Wang H, et al. Pharmacokinetics, tissue distribution, and stability of antisense oligodeoxynucleotide phosphorothioate ISIS 3466 in mice. Oncol Res 1994;6:243-249.

20 Sands H, Gorey-Feret LJ, Cocuzza AJ, et al. Biodistribution and metabolism of internally $3 \mathrm{H}$-labeled oligonucleotides. I. Comparison of a phosphodiester and a phosphorothioate. Mol Pharmacol 1994;45: 932-943.

21 Phillips JA, Craig SJ, Bayley D, et al. Pharmacokinetics, metabolism, and elimination of a 20-mer phosphorothioate oligodeoxynucleotide (CGP 69846A) after intravenous and subcutaneous administration. Biochem Pharmacol 1997;54:657-668.

22 Zhang R, Yan J, Shahinian H, et al. Pharmacokinetics of an anti-human immunodeficiency virus antisense oligodeoxynucleotide phosphorothioate (GEM 91) in HIV-infected subjects. Clin Pharmacol Ther 1995;58:44-53.

23 Zhao Q, Zhou R, Temsamani J, et al. Cellular distribution of phosphorothioate oligonucleotide following intravenous administration in mice. Antisense Nucleic Acid Drug Dev 1998;8:451-458.

24 Liu Z, Carthy CM, Cheung P, et al. Structural and functional analysis of the $5^{\prime}$ untranslated region of coxsackievirus B3 RNA: in vivo translational and infectivity studies of full-length mutants. Virology 1999;265:206-217.

25 Yang D, Wilson JE, Anderson DR, et al. In vitro mutational and inhibitory analysis of the cis-acting translational elements within the $5^{\prime}$ untranslated region of coxsackievirus B3: potential targets for antiviral action of antisense oligomers. Virology 1997;228:63-73.

26 Wang A, Cheung PK, Zhang $\mathrm{H}$, et al. Specific inhibition of coxsackievirus B3 translation and replication by phosphorothioate antisense oligodeoxynucleotides. Antimicrob Agents Chemother 2001;45:1043-1052.

27 Claycomb WC, Lanson Jr NA, Stallworth BS, et al. HL-1 cells: a cardiac muscle cell line that contracts and retains phenotypic characteristics of the adult cardiomyocyte. Proc Natl Acad Sci USA 1998;95: 2979-2984.

28 Chow LH, Gauntt CJ, McManus BM. Differential effects of myocarditic variants of Coxsackievirus B3 in inbred mice. A pathologic characterization of heart tissue damage. Lab Invest 1991;64: $55-64$.

29 Anderson DR, et al. Direct interactions of coxsackievirus B3 with immune cells in the splenic compartment of mice susceptible or resistant to myocarditis. J Virol 1996;70:4632-4645.

30 Mirmomeni MH, Hughes PJ, Stanway G. An RNA tertiary structure in the $3^{\prime}$ untranslated region of enteroviruses is necessary for efficient replication. J Virol 1997;71:2363-2370. 
31 Rohll JB, Moon DH, Evans DJ, et al. The 3' untranslated region of picornavirus RNA: features required for efficient genome replication. J Virol 1995;69: 7835-7844.

32 Xiang W, Harris KS, Alexander L, et al. Interaction between the $5^{\prime}$-terminal cloverleaf and 3AB/3CDpro of poliovirus is essential for RNA replication. J Virol 1995;69:3658-3667.

33 Whelan SP, Wertz GW. Regulation of RNA synthesis by the genomic termini of vesicular stomatitis virus: identification of distinct sequences essential for transcription but not replication. J Virol 1999;73: 297-306.

34 Zheng H, Palese P, Garcia-Sastre A. Nonconserved nucleotides at the $3^{\prime}$ and $5^{\prime}$ ends of an influenza A virus RNA play an important role in viral RNA replication. Virology 1996;217:242-251.

35 Eckstein F. Phosphorothioate oligodeoxynucleotides: what is their origin and what is unique about them? Antisense Nucleic Acid Drug Dev 2000;10:117-121.

36 Crooke ST. Progress in antisense technology: the end of the beginning. Methods Enzymol 2000;313:3-45.

37 Campbell JM, Bacon TA, Wickstrom E. Oligodeoxynucleoside phosphorothioate stability in subcellular extracts, culture media, sera and cerebrospinal fluid. J Biochem Biophys Methods 1990;20:259-267.

38 Phillips MI, Zhang YC. Basic principles of using antisense oligonucleotides in vivo. Methods Enzymol 2000;313:46-56.

39 Kurreck J, Wyszko E, Gillen C, et al. Design of antisense oligonucleotides stabilized by locked nucleic acids. Nucleic Acids Res 2002;30:1911-1918.

40 Aoki M, Morishita R, Higaki J, et al. In vivo transfer efficiency of antisense oligonucleotides into the myocardium using HVJ-liposome method. Biochem Biophys Res Commun 1997;231:540-545.

41 Robaczewska M, Guerret S, Remy JS, et al. Inhibition of hepadnaviral replication by polyethyleniminebased intravenous delivery of antisense phosphodiester oligodeoxynucleotides to the liver. Gene Therapy 2001;8:874-881.

42 Kurreck J. Antisense technologies. Improvement through novel chemical modifications. Eur J Biochem 2003;270:1628-1644.

43 Acsadi G, Jiao SS, Jani A, et al. Direct gene transfer and expression into rat heart in vivo. New Biol 1991;3: 71-81.

44 Gal D, Weir L, Leclerc G, et al. Direct myocardial transfection in two animal models. Evaluation of parameters affecting gene expression and percutaneous gene delivery. Lab Invest 1993;68:18-25.
45 Lin H, Parmacek MS, Morle G, et al. Expression of recombinant genes in myocardium in vivo after direct injection of DNA. Circulation 1990;82: 2217-2221.

46 Krieg AM, Yi AK, Matson S, et al. CpG motifs in bacterial DNA trigger direct B-cell activation. Nature 1995;374:546-549.

47 Krieg AM, Hartmann G, Yi AK. Mechanism of action of CpG DNA. Curr Top Microbiol Immunol 2000;247:1-21.

48 Krieg AM. The role of CpG motifs in innate immunity. Curr Opin Immunol 2000;12:35-43.

49 Krieg AM, Love-Homan L, Yi AK, et al. CpG DNA induces sustained IL-12 expression in vivo and resistance to Listeria monocytogenes challenge. J Immunol 1998;161:2428-2434.

50 Vanderzanden L, Bray M, Fuller D, et al. DNA vaccines expressing either the GP or NP genes of Ebola virus protect mice from lethal challenge. Virology 1998;246:134-144.

51 Knowlton KU, Badorff C. The immune system in viral myocarditis: maintaining the balance. Circ Res 1999;85:559-561.

52 Wessely R, Henke A, Zell R, et al. Low-level expression of a mutant coxsackieviral cDNA induces a myocytopathic effect in culture: an approach to the study of enteroviral persistence in cardiac myocytes. Circulation 1998;98:450-457.

53 Wessely R, Klingel K, Santana LF, et al. Transgenic expression of replication-restricted enteroviral genomes in heart muscle induces defective excitationcontraction coupling and dilated cardiomyopathy. J Clin Invest 1998;102:1444-1453.

54 McManus BM, Chow LH, Wilson JE, et al. Direct myocardial injury by enterovirus: a central role in the evolution of murine myocarditis. Clin Immunol Immunopathol 1993;68:159-169.

55 Zaragoza C, Ocampo C, Saura M, et al. The role of inducible nitric oxide synthase in the host response to Coxsackievirus myocarditis. Proc Natl Acad Sci USA 1998;95:2469-2474.

56 Woodruff JF, Woodruff JJ. Involvement of T lymphocytes in the pathogenesis of coxsackie virus B3 heart disease. J Immunol 1974;113:1726-1734.

57 Huber SA, Pfaeffle B. Differential Th1 and Th2 cell responses in male and female BALB/c mice infected with coxsackievirus group B type 3 . J Virol 1994;68:5126-5132.

58 Huber SA, Moraska A, Choate M. T cells expressing the gamma delta T-cell receptor potentiate coxsackievirus B3-induced myocarditis. J Virol 1992;66: 6541-6546. 